\title{
Hodgkin-Lymphom: Spätfolgen der Bestrahlung abschätzen
}

Ziel dieser Studie war es, die Genauigkeit der aus zweidimensionalen Bestrahlungsplänen rekonstruierten dreidimensionalen Dosisverteilung im Gewebe bei Patienten mit Hodgkin-Lymphom zu beurteilen.

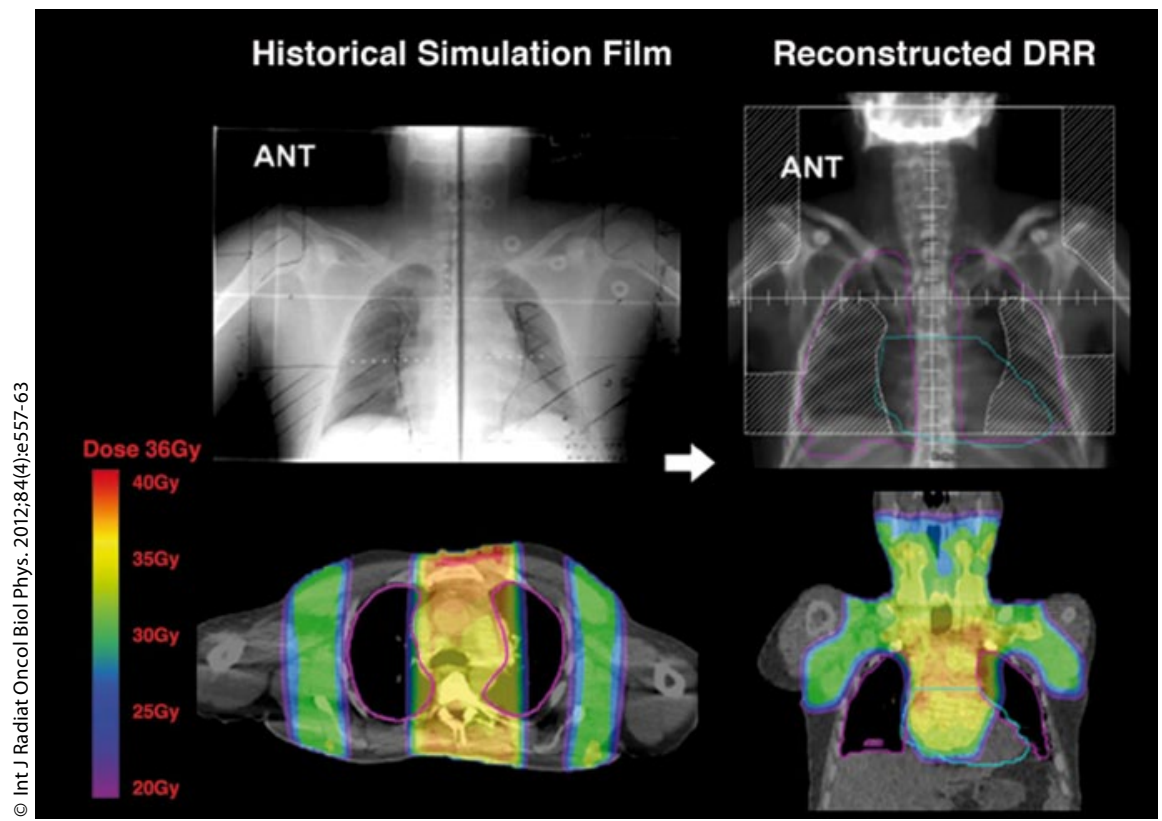

Farbkodierte Dosisverteilung und Dosis-Volumen-Histogramme der rekonstruierten und aktuellen Behandlungspläne eines Patienten. Durch die Rekonstruktion können die absorbierten Dosen noch Jahre später genau berechnet werden.

$\mathrm{m}$ effektivere Strahlentherapien $\mathrm{zu}$ entwickeln, muss man den Zusammenhang zwischen normalen Gewebedosen und später Bestrahlungstoxizität verstehen. Bisher sind Langzeitergebnisse jedoch im Allgemeinen nur für Pati- enten verfügbar, die sich einer zweidimensionalen Behandlungsplanung unterzogen haben. Für Dosis-Risiko-Modelle kann man aus zweidimensionalen Bestrahlungsplänen die dreidimensionale Dosisverteilung rekonstruieren (Abb.).
In der vorliegenden Studie geschah dies für Patienten mit Hodgkin-Lymphom, die mediastinal bestrahlt worden waren. Dabei wurden die Volumina von Lunge, Herz und Brust aus den zweidimensionalen Bestrahlungsplänen dreidimensional rekonstruiert. Anschließend wurde für jedes rekonstruierte Organ eine mittlere Dosis und ein mittleres Volumen berechnet, das von mindestens 5 Gy $\left(V_{5}\right)$ bzw. 20 Gy $\left(V_{20}\right)$ erreicht wurde. Dieses Verfahren wurde bei 15 Patienten durchgeführt, für die es sowohl 2D- als auch 3D-Bestrahlungspläne, sowie für zehn Patienten, für die es lediglich eine 2D-Bildgebung gab.

Die Auswertungen bei Patienten mit 3D-Bestrahlungsplänen ergaben, dass die normale Gewebedosis anhand der 2D-Bestrahlungspläne genau rekonstruiert werden kann. Sie waren bei den alten Mantelfeld-Bestrahlungen wesentlich höher als bei der mediastinalen InvolvedField-Bestrahlung: 15,2 Gy vs. 10,6 Gy (Lunge), 27,0 Gy vs. 14,3 Gy (Herz) und 8,0 Gy vs. 3,2 Gy (Brust).

Fazit: Organ-Dosen können anhand von 2D-Daten noch viele Jahre nach Exposition genau dreidimensional berechnet werden. Damit ließe sich der Zusammenhang zwischen dreidimensionaler Dosisverteilung im Gewebe und späten Strahlenfolgen quantifizieren. Judith Neumaier

$\mathrm{Ng}$ A et al. Individualized 3D reconstruction of normal tissue dose for patients with long-term follow-up: A step toward understanding dose risk for late toxicity. Int J Radiat Oncol Biol Phys. 2012;84(4):e557-63.

\section{Weitere Infos auf springermedizin.de}

Lesen Sie mehr zum Thema "Non-Hodgkin-Lymphome" im Dossier. Die Erkrankungen, die unter dem Oberbegriff Non-Hodgkin-Lymphom zusammengefasst werden, unterscheiden sich hinsichtlich Ursache und Manifestation stark. Die Diagnostik und Therapie stellt deshalb eine besondere Herausforderung für den Onkologen dar.

Unter anderem: „T-Zellen im FokusImmuntherapie: Heilung bei aggressiven Tumoren in Sicht." Mit neuen Immuntherapeutika werden überraschende Erfolge erzielt; bei Melanomen aber auch bei Lymphomen. www.springermedizin.de/4323202

Non-Hodgkin-Lymphome (NHL) einschließlich der großzelligen anaplastischen Lymphome sind bei Kindern und Jugendlichen etwas häufiger als das Hodgkin-Lymphom. Prüfen Sie Ihr Wissen im CME-Beitrag "Non-Hodgkin-Lymphome bei Kindern und Jugendlichen - Von der Diagnostik bis zur Nachsorge" www.springermedizin.de/eAkademie 